\title{
Charging transient in polyvinyl formal
}

\author{
P K KHARE*, P L JAIN ${ }^{\dagger}$ and R K PANDEY ${ }^{\ddagger}$ \\ Department of Postgraduate Studies \& Research in Physics \& Electronics, Rani Durgavati University, \\ Jabalpur 482 001, India \\ ${ }^{\dagger}$ Department of Physics, Government PG College, Damoh 470 661, India \\ ${ }^{\ddagger}$ Department of Physics, Dr HS Gour University, Sagar 470 002, India
}

MS received 14 August 2000; revised 2 May 2001

\begin{abstract}
In the present paper charging and discharging transient currents in polyvinyl formal (PVF) were measured as a function of temperatures $\left(40-80^{\circ} \mathrm{C}\right)$, poling fields $\left(9.0 \times 10^{3}-9.0 \times 10^{4} \mathrm{~V} / \mathrm{cm}\right)$ and electrode combinations (Al-Al, Au-Al, $\mathrm{Zn}-\mathrm{Al}, \mathrm{Bi}-\mathrm{Al}, \mathrm{Cu}-\mathrm{Al}$ and $\mathrm{Ag}-\mathrm{Al}$ ). The current-time characteristics have different values of slope lying between $0.42-0.56$ and 1.42-1.63. The polarization is considered to be due to dipolar reorientation associated with structural motions and space charge relaxations due to trapping of injected charge carriers in energetically distributed traps.
\end{abstract}

Keywords. Charging transient currents; polyvinyl formal; dipolar mechanism; space charge relaxations.

\section{Introduction}

Transient charging and discharging currents were investigated to understand time dependent polarization effect in organic polymers (Dasgupta and Joyner 1976; Vanderschueren and Linkens 1978; Neagu and Neagu 1994). The polarization of polymeric materials may be due to dipolar orientation and accumulation of charge carriers near the electrodes or trapping in the bulk (Pillai et al 1983). Under certain conditions additional charge carriers may be provided by injection from the electrodes, which also contribute space charge polarization (Lindmayer 1965; Taylor and Lewis 1971; Wintle 1975; Dasgupta and Broklay 1978; Khare and Singh 1994; Gour et al 1995). Hopping of charge carriers from one localized state to another (Jonscher 1972) and tunneling from electrode to those trap levels in the dielectric which are close to the Fermi level of the electrode material (Wintle 1973) have also been proposed to account for the currents in the dielectrics.

The current obtained immediately after the application of a step function voltage is known in most cases to decay with time until a steady state current is achieved (Lakshmi Narayana et al 1994). The discharging current flowing on removal of the voltage is usually the mirror image of the charging current except that a steady state current does not occur. The knowledge of currents is also necessary to discover the true conductivity of the material (Pillai and Rashmi 1981). Generally. it is expected that the transient and steady state conductivities should have similar cha-

\footnotetext{
*Author for correspondence
}

racteristics or mechanisms. But it is not absolutely necessary and they may have different mechanisms inspite of having similar activation energies for both the conductivities.

The mechanisms of the time-dependent polarization effects may be studied from an analysis of transient currents in charging and discharging modes with respect to polarizing fields, temperatures and electrode materials. In continuation of our previous work (Khare et al 1995; Khare 1996; Khare and Jain 1997), the present work reports the results of such studies with PVF films.

\section{Experimental}

Polyvinyl formal (PVF) (molecular weight 50,000) films of thicknesses about $20 \mu \mathrm{m}$ were prepared by the solution grown technique. A $8 \%$ PVF solution was prepared by dissolving $2.4 \mathrm{~g}$ of PVF in $30 \mathrm{ml}$ of solvent, consisting of a mixture of benzene and methanol in the ratio $3: 2$. The solution was continuously stirred for about $40 \mathrm{~min}$ by means of a teflon coated magnetic stirrer and heated to $60^{\circ} \mathrm{C}$ to ensure homogeneous mixing of the solvent. Films were grown on the surface of cleaned microscopic glass slides. Ultrasonically cleaned vacuum metallized microscopic glass slides were immersed vertically into the solution. Films were grown by slowly pulling the slides out vertically from the solution. The time of immersion and the concentration of the solution determined the film thickness. After deposition of the film, the glass slides were taken out and dried in an oven at $50^{\circ} \mathrm{C}$ for $24 \mathrm{~h}$. This was followed by room temperature outgassing at $133.33 \times 10^{-5} \mathrm{Nm}^{-2}$ for a further period of $24 \mathrm{~h}$. The film thickness was determined capacitively, taking the diele- 
ctric constant of PVF as 3.7 (Bandrup and Immergut 1975).

In the present study, the samples were thermally poled with fields of $9.0 \times 10^{3}-9.0 \times 10^{4} \mathrm{~V} / \mathrm{cm}$ at various temperatures (ranging from $40-80^{\circ} \mathrm{C}$ ) for $100 \mathrm{~min}$ during which the transient current in the charging mode was observed $2 \mathrm{~min}$ after the application of the field. The measurements were carried out in a suitable cell using an assembly which was dry, rigid and well established. This assembly had the following properties: (i) high insulating resistance, (ii) freedom from picking up of spurious potentials and induced charges due to physical motion of the system, and (iii) negligible leakage current and less microphonic. To achieve the required degree of precision every consideration of perturbing parameter was taken into account, while designing the electrode assembly. Also, while carrying out measurements proper care was taken to avoid stray currents that may affect the results. The polarization was carried out by connecting a d.c. power supply (EC-HV 4800D) in series with an electrometer 600B which was carefully shielded and grounded to avoid ground loops and extraneous electrical noise. The electrometer was specially designed to measure very small direct current (ranging from $10^{-6}$ to $10^{-14}$ amp), low d.c. potentials from high impedance source, small charges and high resistance.

\section{Results and discussion}

Figure 1 shows typical charging currents vs time characteristics for an applied field of $3.6 \times 10^{4} \mathrm{~V} / \mathrm{cm}$ for temperatures $40,50,60,70,75$ and $80^{\circ} \mathrm{C}$. The discharging currents vs time curves (dotted lines) for 40, 50, 60,70 and $80^{\circ} \mathrm{C}$ have been placed on the same figure. It is evident from the figure that the initial slope of the charging current curves decreases with the rise of temperature. The discharging current plots are entirely different from those of charging currents. The difference between charging and discharging currents increases with the rise of temperature. The field dependence of the charging currents at $80^{\circ} \mathrm{C}$ is shown in figure 2 . The charging current vs time characteristics consisted of two slopes for each field. The initial slope of the curve decreases with the increase in applied field. The curve has a knee which shifts towards lower values of time with the increase in field. Figure 3 shows the electrode effect on charging currents at $80^{\circ} \mathrm{C}$ for an applied field of $3.6 \times 10^{4} \mathrm{~V} / \mathrm{cm}$. The charging current was maximum for $\mathrm{Au}-\mathrm{PVF}-\mathrm{Al}$ system, while it was minimum for Al-PVFAl system. The decay of the charging current is very fast for $\mathrm{Au}-\mathrm{Al}$ pair, while it is slow for $\mathrm{Bi}-\mathrm{Al}$ pair. All these curves are approximately parallel i.e. having similar decaying nature at higher values of time. The field dependence of isochronal currents (i.e. the charging current at a particular time) are plotted at $80^{\circ} \mathrm{C}$ for 2,6 and $10 \mathrm{~min}$. The current-field characteristics were observed to become gradually superlinear at higher fields.

The transient current flowing through a dielectric after the application or removal of a step voltage decays logarithmically following the Curie-von Schweidler law

$$
I(t)=A(T) t^{-n},
$$

where $I$ is the current, $A(T)$ the temperature-dependent factor, $t$ the time after application or removal of the external voltage and $n$ a constant which is generally close to unity.

The current, in the time domain, for the short time region is characterized by the relation

$$
I(t) \propto t^{-n} ; \quad 0<n<1 ; \quad t \ll \frac{1}{W_{\mathrm{p}}},
$$

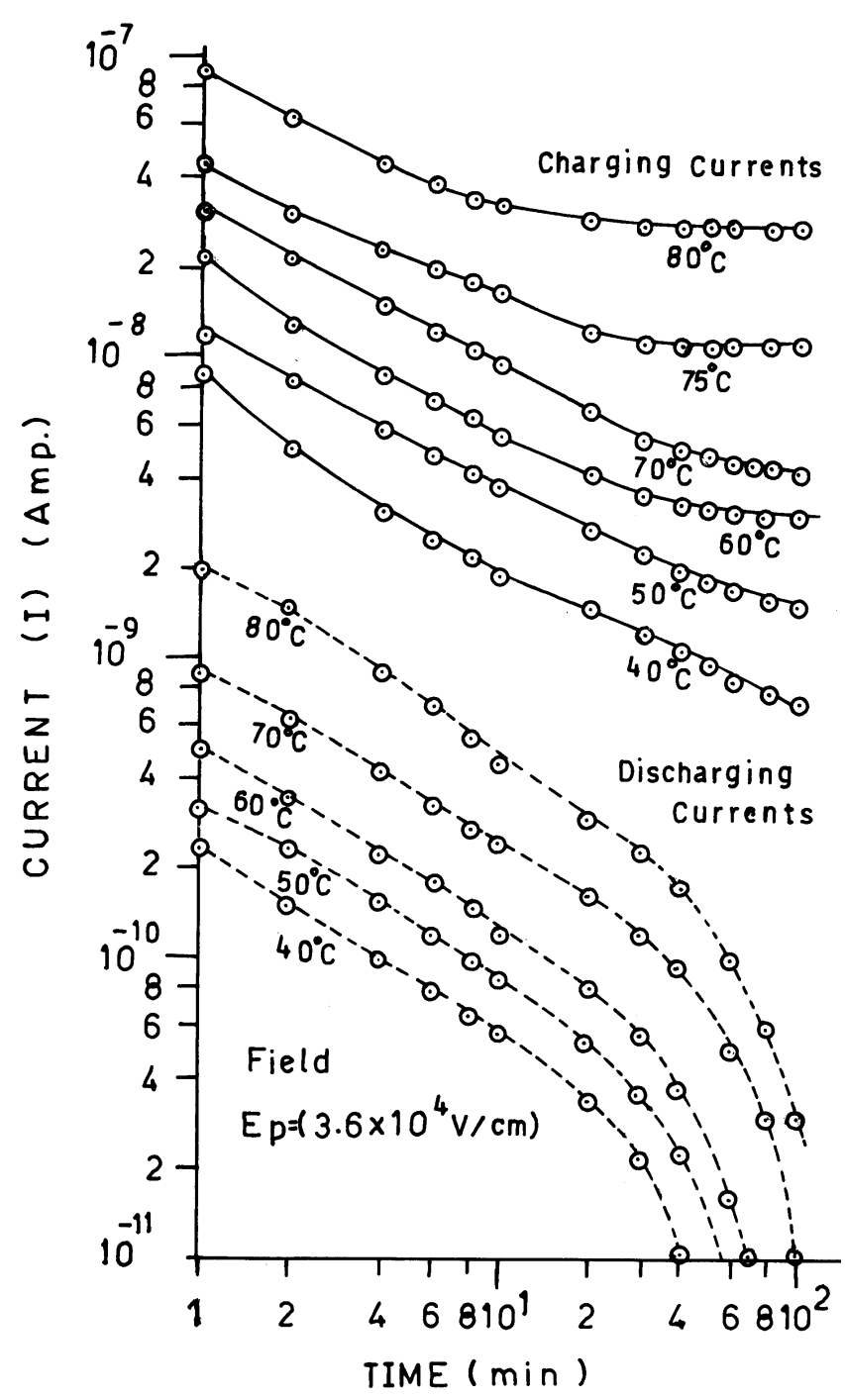

Figure 1. Charging and discharging transient characteristics of PVF films at various temperatures (i.e. 40, 50, 60, 70, 75 and $\left.80^{\circ} \mathrm{C}\right)$ with constant poling field $\left(E_{\mathrm{p}}=3.6 \times 10^{4} \mathrm{~V} / \mathrm{cm}\right)$. 
i.e. at frequencies which are larger than the loss peak frequency $W_{\mathrm{p}}$, and for long time region

$$
I(t) \propto t^{-1-p} ; \quad 0<n<1 ; \quad t \gg W_{\mathrm{p}},
$$

with logarithmic slope steeper than unity. The two power losses determine the time domain response of dipolar system. Similar behaviour is observed in carrier dominated systems, however, low frequency dispersion below a frequency $W_{\mathrm{c}}$, which corresponds to long time region is described by the power laws with small value of $n$. Let $n=1-p$, with $p$ close to unity, for low frequency dispersion regime.

The long time response of charge carrier system will then be denoted by

$$
I(t) \propto t^{-1-p} ; \quad p=1 ; \quad t \gg \frac{1}{W_{\mathrm{p}}},
$$

which corresponds to a very slowly time varying current.

The complete representation of the universal dielectric response in the time domain, covering both dipolar loss peaks and strong low frequency dispersion associated

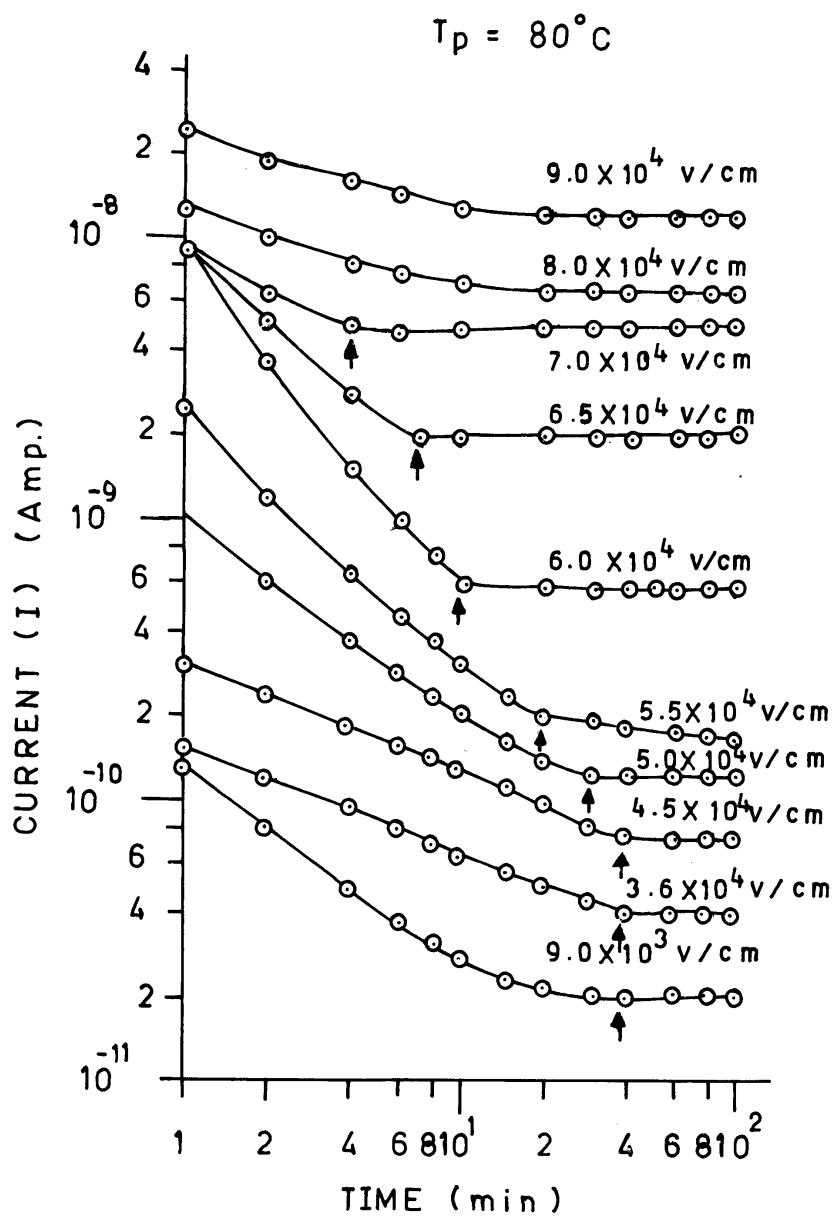

Figure 2. Charging characteristics of PVF films with various poling fields at constant temperature $\left(T_{\mathrm{p}}=80^{\circ} \mathrm{C}\right)$. with the charge carrier dominated system may be represented by

$$
I(t) \propto t^{-n} ; \quad 0<n<2,
$$

with the exponent $n$ taking values in different ranges at long and short times, respectively (Khare et al 2000). We are doing transient current measurements and as such quantitative variation of $A(T)$ is not seen.

At temperatures much lower than the glass transition temperatures and for the low to moderate fields used, several of the various concepts previously postulated to account for the transient conduction phenomena in polymers can be ruled out on the basis of the experimental facts (Vanderschueren and Linkens 1978). Tunneling can be unequivocally eliminated as a possible mechanism since, in most cases the currents are strongly temperature dependent. So far as the nature of trapping sites are concerned, it is generally conceded that charge trapping is primarily due to the basic polymer structure which can be of various traps. This, one can envisage physical traps in cavities due to defects and free volume inherent in the bulk polymer structure, the binding energy resulting from a polarization of the surrounding molecules. Another type of trap or hopping site may be due to chemical heterogeneities in the polymer structure (Khare 1996). Although

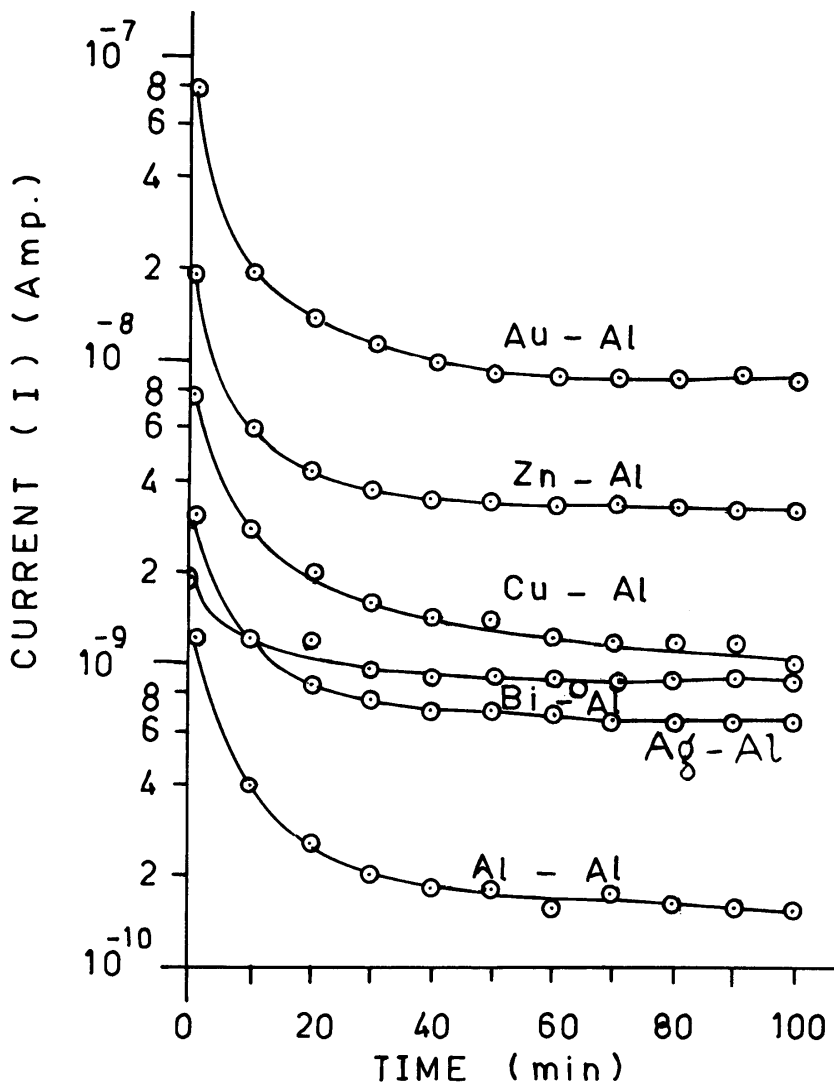

Figure 3. Electrode effect on charging currents at $80^{\circ} \mathrm{C}$ with constant poling field $\left(E_{\mathrm{p}}=3.6 \times 10^{4} \mathrm{~V} / \mathrm{cm}\right)$. 
the dielectric response is commonly associated with orientation of permanent dipoles, it is undesirable that hopping charges of either electronic or ionic nature may give rise to a very similar dielectric behaviour. The important distinction lies in the degree of localization of these carriers (Singh et al 1984). An electron, or an ion, confined to hopping between two preferred positions is indistinguishable from a dipole, while a distinctly different situation arises where the carrier is free to execute hops over finite paths, some of which may eventually extend all the way from one electrode to the other.

The magnitude of charging currents is greater than the discharging currents at the corresponding temperatures (figure 1). It appears that charge involved in the charging period is greater than the discharge period. The possible reason for this difference may be that the initial transients occur with empty traps and currents will be as large as allowed by the injecting barriers. As the traps become filled, the current reduces to the space charge limited current with traps. In the discharging period, on the other hand, the trapped carriers will be discharged towards both electrodes showing a smaller current in the external circuit. The Curie-von Schweidler law was not found to be obeyed for a longer period of time and the latter becomes progressively shorter (i.e. more and more limited period of time) with the increase in temperature. The transient charging and discharging currents are not the mirror images of each other (in any respect up to any extent) which is the essential condition for tunneling, dipole or electrode polarization process. The value of $n$ for the discharge current was observed to be less than 1 and to decrease with the rise of temperature. This can be observed by the decreasing slope of the initial discharging current, i.e. at lower values of time but for longer periods of time, the value of $n$ becomes greater than 1 .

The time-dependent charging current of PVF sample at $80^{\circ} \mathrm{C}$ for various values of electric fields are shown in figure 2. The application of a step voltage to a dielectric causes a flow of current which decays with time before reaching a steady state value. The total current (Lyschekin 1984) consisting of the absorption current $\left(I_{\mathrm{a}}\right)$ and the static current $\left(I_{\mathrm{s}}\right)$, i.e.

$$
I=I_{\mathrm{a}}+I_{\mathrm{s}}
$$

For any given electric field, the time dependence of transient current is found to obey the law of dielectric responses (Aguilar et al 1989). Soon after the application of the poling field in a metal-insulator-metal system, the current rises rapidly and then approaches a constant value. We have to consider four components of the current in such a system: (i) the current controlled by various polarization mechanisms; (ii) the current controlled by the charging of the capacitor through a resistor, $R$; (iii) the space charge limited current, and (iv) the conduction current which is time dependent. The former components gradually fall off to zero within a hundredth of a second. The third component is due to formation of space charge. The residual former current is referred to as bulk current, which may be ionic, electronic or both (Khare et al 2000). In the present study, the large currents obtained just after the application of voltage, subsided to much smaller steady values after a certain length of time. This is because as the total current is the sum of absorption currents which decays with time and static current which remains constant. The absorption current reduces to zero when the sample is completely polarized. From figure 2 it is observed that the rate of fall of the absorption current is low for lower electric fields than for higher fields, i.e. at higher fields the current approaches a stable value in a relatively shorter time after the field is applied. It means that the polarization time decreases with the increase of electric field, suggesting a dependence of the resistance of the film on the level and duration of applied voltage. The time-dependent absorption current suggests that the polarization in sample may be due to dipolar orientation and trapping of charge carriers in the bulk, whose injection from the traps will increase with increasing field and temperature. The sudden application of voltage causes a cloud of carriers, i.e. a space-charge, to be injected from the contact into the sample. This free charge gives rise to a large burst of current. However, one must take into account the effects of trap densities in the sample. The free charge forced into the sample settles into the traps and one observes the decay of current, the rate being determined by the capture cross-section of traps for free carriers. The appearance of knee can be expected to be due to the onset of a quasi-steady state conduction in a shorter time. The shifting of the knee is also due to the progressive superposition of the current growing exponentially.

The charging currents in the present study are markedly affected by the electrode materials (figure 3) and this can be explained in terms of charge injection as a probable mechanism. The primary source of charge carriers is the electrode contact, a potential difference of work function $\phi_{2}-\phi_{1}$, is created between the two metals. The semiinsulating polymer differs from a metal, however, in that an electric field may exist within the interior of the semiinsulator. For this reason the contact potential drop between the metal and the semi-insulator may take place within the material rather than at the contact interfaces. Alongwith the field, there may exist a depletion in the accumulation of charges in the surface layers. The work function of the polymeric material is higher compared to that of the metals used for contacts, which creates an accumulation layer at the interfaces and leaves charged donors in the bulk. This in turn creates fields at the interfaces of the metal-polymer system (Khare et al 1994). Let us suppose an insulator has a large energy gap with its Fermi level located above the mid gap and there is no surface state; then after contact with a metal surface, the 


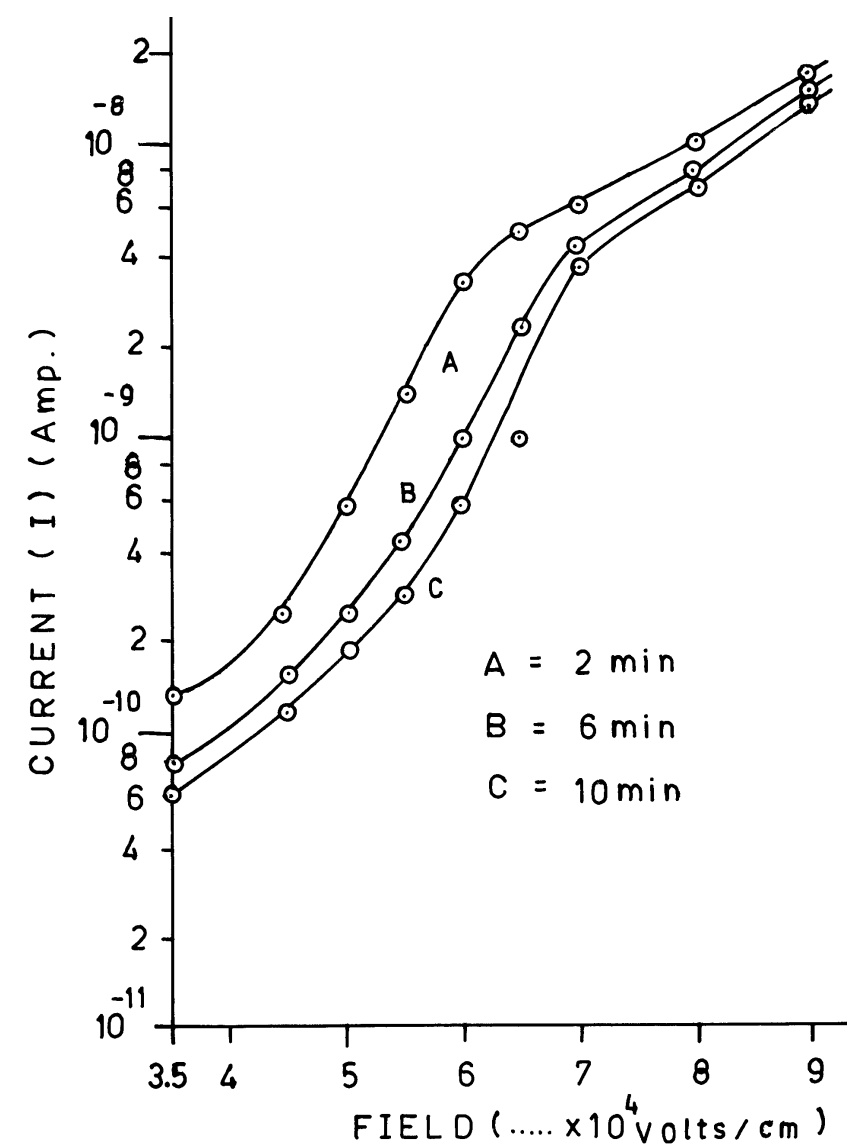

Figure 4. Field dependence of isochronal characteristics for 2, 6 and $10 \mathrm{~min}$ at $80^{\circ} \mathrm{C}$.

electron transfer at contact equalizes both Fermi levels. The barrier height depends on the relative values of metal work function and the electron affinity of the insulator (Takai et al 1975). The surface state is one of the factors which affect injection phenomena. Donor-like surface states located between the conduction band and the Fermi level, after contact tends to bend the band edge towards the Fermi-level, making electron injection from the metal much easier. On the other hand, acceptor like surface states located between the valence band and Fermi-level act as a stepping-stone for electrons injected from the valence band of the insulator to the metal, thus enhancing the hole injection (Khare et al 1997). The field dependence of isochronal charging current is shown in figure 4 . The current-field characteristics were observed to become gradually superlinear at high fields. The superlinear behaviour supports the theory of charge injection forming trapped space charges.

As PVF is a weakly polar polymer (the dipolar group is rigidly attached to the main chain of PVF), the probability of charge carriers are present in it. The charging currents observed in PVF is expected to show dipolar relaxation behaviour. The linear region in the charging current vs poling field curves (figure 2) at lower fields seems to favour internal dipolar polarization. However, the possi- bility of weak carrier injection at such field values cannot be ruled out. The injected charges are present at different trapping sites leading to a space charge which fundamentally influences all the transport phenomena and their effects at the electrodes.

The different facts, including the weak polar structure of polymer, the power-law dependence of current on field, the observed value of ' $n$ ' and the thermal activation of current over a certain temperature range indicate that a space charge due to accumulation of charge carriers near the electrodes and trapping in the bulk may account for the observed current. The decay of current in the longer time region for different samples indicates the existence of energetically distributed localized trap levels. As temperature increases, the mobility of the carriers also increases, hence all the deeper traps are filled. Release of a large number of charge carriers from the traps during experiment may then lead to blocking of electrodes, causing a decrease in current. Charge injection from the electrodes, with subsequent trapping of injected charges in or near surface region gives rise to homospace charge and the thermal release of charge carriers from the traps. Before a trapped space charge injected at higher fields is thermally released, a space charge barrier is present at the electrodes, which suppresses the entrance of charge carriers into the sample. Thus, the observed current remains smaller.

\section{Conclusions}

The decaying trends of currents with time, the superlinear nature of the isochronal currents against fields, the electrode dependence nature of the transient current seems to be sufficient and reliable ground to assume that the possible controlling mechanism for the transient phenomenon is the charge injection and formation of trapped charges.

\section{References}

Aguilar M, Jaque F and Batta-Calleja F J 1981 J. Mater. Sci. Lett. 162079

Bandrup J and Immergut E H 1975 Polymer handbook (NY: John Wiley)

Dasgupta D K and Joyner K 1976 J. Phys. D 9829

Dasgupta D K and Broklay R S 1978 J. Phys. D 11955

Gour M S, Reeta Singh, Khare P K and Singh Ranjeet 1995 Polym. Int. 3733

Jonscher A K 1972 J. Non-Cryst. Solids 8293

Khare P K 1996 Pramana - J. Phys. 46109

Khare P K and Singh Ranjeet 1994 Polym. Int. 34407

Khare P K and Jain S K 1997 Indian J. Pure and Appl. Phys. 35 408

Khare P K, Chandok R S, Dubey N and Srivastava A P 1994 Polym. Int. 35153

Khare P K, Chandok R S and Srivastava A P 1995 Indian J. Phys. 69627 
Khare P K, Kuraria R K and Jain S K 1997 J. Polym. Mater. 14 133

Khare P K, Pandey R K, Chourasia R R and Jain P L 2000 Polym. Int. 49719

Lakshmi Narayana K, Dasaradhudu Y and Narsimha Rao V V R 1994 Polym. Int. 35315

Lindmayer J 1965 J. Appl. Phys. 36196

Lyschekin G A 1984 Polymer electrets (Moscow, USSR) p. 81

Neagu E R and Neagu R M 1994 Phys. Status Solidi 144429

Pillai P K C and Rashmi 1981 Eur. Polym. J. 17611
Pillai P K C, Narula G K, Tripathi A K and Mendiratta R G 1983 Phys. Status Solidi 77693

Singh Ranjeet, Dasgupta N and Yadav L P 1984 Indian J. Pure \& Appl. Phys. 22222

Takai Y, Osawa T, Chikao K, Mizutani T and Ieda M 1975 Jpn. J. Appl. Phys. 10473

Taylor D M and Lewis T J 1971 J. Phys. D 41346

Vanderschueren J and Linkens A 1978 J. Appl. Phys. 494195

Wintle H J 1973 J. Appl. Phys. 442514

Wintle H J 1975 Solid State Electron. 181039 